\title{
ESTUDO COMPARATIVO DO EFEITO DE SOLUÇÃO DE NaF A 0,5\%, ATRAVÉS DE BOCHECHO, ESCOVAÇÃO E AMBOS, NA PREVENÇÃO DA CÁRIE DENTAL *
}

\begin{abstract}
CASTELlanos, R. A. Estudo comparativo do efeito de solução de NaF a $0,5 \%$, através de bochecho, escovação e ambos, na prevenção da cárie dental. Rev. Saúde públ., S. Paulo, 17:461-75, 1983.

RESUMO: Foi realizado estudo a fim de comparar o efeito na redução do ataque de cárie de uma solução de fluoreto de sódio, na concentração de $0,5 \%$ aplicada sob a forma de bochecho, escovação, escovação e bochecho, uma vez por semana, sob supervisão, durante um período escolar de 10 meses em 424 escolares de 9 a 10 anos de idade matriculados em cinco escolas estaduais do Estado de São Paulo (Brasil). As crianças foram distribuídas igualmente em três grupos experimentais e um grupo controle, de acordo com a idade dental ( $\mathrm{n}^{\circ}$ de superfícies de dentes permanentes irrompidas) e experiência anterior de cárie (CPO-S). Foram examinadas 307 crianças e os resultados mostraram que houve uma redução, estatisticamente significante ao nível de 0,05 , de ataque de cárie entre os grupos experimentais e controle, da ordem de $35,19 \%$ para o grupo de bochecho (grupo I), de $37,91 \%$ para o grupo de escovação (grupo II), e de $49,77 \%$ para o grupo de escovação e bochecho (grupo IV). Quando comparados os grupos experimentais entre si não houve diferenças estatisticamente significantes ao nivel de 0,05 .
\end{abstract}

UNITERMOS: Cárie dentária, prevenção. Flúor. Escolares.

\section{INTRODUÇAO}

As soluções de flúor aplicadas em forma de bochechos ou por meio de escovaçōes têm recebido muita atenção nas últimas décadas; seu uso como medida de prevenção é um fato comprovado por vários autores ${ }^{3,4,27,33,55}$ e recomendado pela Organização Mundial da Saúde ${ }^{43}$ para serem utilizadas no campo da odontologia preventiva. Não obstante ter sido demonstrada sua eficácia, sua utilização em saúde pública ainda é limitada.

Os estudos sobre as soluções de flúor variam quanto ao tipo de solução empre- gada, quanto à sua concentração, seu $\mathrm{pH}$ e quanto à freqüência de sua utilização. Da mesma forma, variam as idades das crianças que participaram dos experimentos, sendo que a maioria dos autores tem utilizado crianças em idade escolar, predominando entre estas as de 10 a 13 anos $15,22,25,27,33,45,46,48,55$.

A solução de flúor empregada com maior frequiência tem sido a de $\mathrm{NaF}$ a $0,2 \% 2,77,9,20,27,33,35,47,53,55$.

Alguns pesquisadores tem utilizado solução de flúor-fosfato neutro ou acidu-

* Resumo parcial da Tese de Doutorado apresentada à Faculdade de Saúde Pública da Univerisidade de São Paulo, em 1979, subordinada a este mesmo título.

* Do Departamento de Prática de Saúde Pública da Faculdade de Saúde Pública da USP - Av. Dr. Arnaldo, 715 - 01255 - São Paulo, SP - Brasil. 
CASTELLANOS, R.A. Estudo comparativo do efeito de solução de NaF a $0,5 \%$, através de bochecho, escovação e ambos, na prevenção da cárie dental. Rev. Saúde públ., S. Pailo, $17: 461-75,1983$.

lado $1,6,18,21,46$ e outros solução de fluoreto de estanho $34,45,52$, ou ainda outros tipos de soluçōes $15,23,2: 9,5 \pi$.

Outros estudos foram realizados usancio-se a escováą̧a como método de aplicaçāo das soluções de flúor $3,4,10,11,4,3,26,28,42,47$.

Berggren e Welander ${ }^{4}$, num estudo de dois anos, usaram solução de $\mathrm{NaF}$ a $1 \%$, no qual $y$ escovações foram feitas obtendo redução de 25 a $30 \%$ no incremento da cárie; os mesmos autores ${ }^{3}$ levaram a cabo outro estudo, utilizando 3 diferentes soluções de flúor; uma delas foi o $\mathrm{NaF}$ a $0,5 \%$ que, aplicado cinco vezes ao ano, deu uma reduçāo de $29 \%$ na incidência da cárie dental.

Ollinen 42 não encontrou diferenças significantes entre bochechos e escovações com uma solução de $\mathrm{NaF}$ a $0,5 \%$, aplicada mensalmente e por um período de 3,5 anos.

Rosenkranz ${ }^{47}$, também, comparou os métodos de escovação e bochechos, com solução de $\mathrm{NaF}$ a $0,2 \%$, aplicada uma vez cada duas semanas; após um ano de estudo a diferença entre os grupos não foi significante.

Bullen e col.10,11, Conchie e col.13 e Heifetz e col. ${ }^{26}$ utilizaram solução ou gel de flúor-fosfato acidulado, com resultados que variaram respectivamente de $38,5 \%$, $15 \%, 25 \%$, e nenhuma redução de superfícies com experiência de cárie.

No Brasil, poucos dados são disponíveis a respeito do emprego de soluções de flúor em forma de bochechos ou escovações.

Moreira ${ }^{39}$ realizou um experimento, usando uma solução molar de $\mathrm{NaF}$ a $0,1 \%$, no qual as crianças que bochecharam 3 vezes por semana, durante um ano, obtiveram uma redução significante na incidência da cárie dentária de $51,1 \%$.

Horowitz e col.28, num outro estudo realizado em São Paulo, usando flúor-fosfato em forma de gel, após 3 anos, durante os quais 15 escovações foram feitas, obtiveram uma redução de $26 \%$ na experiência de cárie dental.
Ao analisarmos as pesquisas realizadas com bochechos e escovações a base de flúor, verificamos faltar estudos que comparem os efeitos de bochechos e escovações, usados independentemente e em conjunto, realizados uma vez por semana, com solução de flúor.

O objetivo da presente investigação foi verificar, após um ano de estudo, a eficácia de uma solução de $\mathrm{NaF}$ a $0,5 \%$, aplicada em escolares, em formá de bochechos, escovações e escovação e bochecho.

\section{MATERIAL E METODOS}

\section{Amostra}

Constou de 424 crianças de 9 e 10 anos de idade, matriculadas em 5 escolas da rede estadual da cidade de São Paulo, no ano de 1975 .

As crianças foram distribuidas igualmente, de acordo com o número de superfícies irrompidas e de acordo com a experiência anterior de cátie (CPO-S médio), em um grupo controle e em 3 grupos experimentais.

O tamanho de amostra foi calculado de acordo com o método de Marthaler ${ }^{36}$, considerando os seguintes pressupostos:

- redução da incidência da cárie de aproximadamente $30 \%$; coeficiente de variação $(S / \bar{\chi})$ iguál a 1,0 e nivel de significância de 0,05 , sendo o desvio padrão constante, isto $\dot{e}$, igual tanto para o grupo controle como para os grupos experimentais, e perda de escolares de aproximadamente $20 \%$.

Assim, cada um dos 4 grupos ficou constituído por 106 crianças, de ambos os sexos, sendo um controle (grupo III), que não realizou o bochecho nem a escovação com solução alguma.

- Grupo I: crianças que realizaram bochechos semanais com a solução de $\mathrm{NaF}$ a $0,5 \%$ por um min, 
CASTELLANOS, R.A. Estudo comparativo do cfeito de solução de NaF a $0,5 \%$ através de bochecho, escovaçāo e ambos, na prevencāo da cárie dental. Rev. Saúde públ., S. Paulo, 1\% : $461-75,1983$.

- Grupo 11: crianças que realizaram uma escovação semanal com a solução de $\mathrm{NaF}$ a $0,5 \%$ durante $3 \mathrm{~min}$, e

- Grupo IV: crianças que realizaram semanalmente primeiro a escovação, com a solução de $\mathrm{NaF}$ a $0,5 \%$, e depois efetuaram o bochecho, com a duração de 3 e um min respectivamente para a escovação e para o bochecho.

Registro: As informações relativas ao nome, sexo, idade e local de nascimento foram retiradas do boletim escolar. Os dados de identificação das crianças, os exames clínicos e os dados sobre bochechos e escovações foram registrados em fichas apropriadas. Os exames clínicos foram feitos com o auxílio de espelhos bucais planos, sondas exploradoras no 5 e luz natural.

Indices: foi utilizado o indice CPO-S proposto em 1938 por Kleiso e foram seguidos os critérios recomendados pela Disciplina de Odontologia Sanitária da Faculdade de Saúde Pública da Universidade de São Paulo ${ }^{17}$. A calibração para esta pesquisa foi satisfatória pois as discordâncias foram menores que $5 \%$.

Preparo e aplicação da solução NaF a 0,5\%: o sal foi pesado em balança analitica marca Microwa, por um técnico do Departamento de Nutrição da Faculdade de Saúde Pública da USP e acondicionado em doses individuais de $5 \mathrm{~g}$. Momentos antes de sua utilização as soluçōes de flúor eram preparadas com um litro de água potável e $5 \mathrm{~g}$ de $\mathrm{NaF}$ e agitadas até a dissolução completa do pó.

A orientação e a supervisão dos bochechos e das escovaçōes foi realizada por cirurgiões-dentistas.

As crianças foram ensinadas e treinadas a executar as técnicas de escovação e de bochechos em duas sessões prévias à execução definitiva, utilizando-se água potável em lugar da solução de flúor.

Procurou-se obter autorização e envolver os professores e pais de alunos no desen- volvimento da pesquisa com o objetivo de que incentivassem as crianças a executar os procedimentos e a seguir as instruçōes para a utilização adequada da técnica. Para tanto foram realizadas reunióes de pais e mestres nas quais foram expostos os objetivos da pesquisa, a importância da participação continuada das crianças e os beneficios da implantação de programas sistemáticos de aplicação de flúor.

Técnica de bochecho: Proporcionou-se a cada estudante um copo com $10 \mathrm{ml}$. da solução de flúor; com essa quantidade os escolares realizaram um bochecho durante um minuto, fazendo com que a solução entrasse em contato com todas as superficies dentárias. Depois dos bochechos as crianças voltaram às suas classes, com a instrução de não beberem líquidos (água, sucos, etc.) e nem comerem alimentos pelo espaço de, no mínimo, 30 mín.

Parć facilitar a ministração e a supervisão dos bochechos, foram chamados, de cada vez, grupos de 10 crianças.

Técnica de escovação: a escovação foi feita por segmentos, sendo 4 posteriores e 2 anteriores, e nesta ordem: segmento póstero-superior direito (área de molares e pré-molares); segmento ântero-superior (canino a canino); segmento póstero-superior esquerdo; segmento póstero-inferior esquerdo; segmento ântero-inferior $\mathrm{e}$ terminando com o segmento póstero-inferior direito, com o último dente distal ao canino inferior direito.

A sequiencia da técnica de escovação das superficies dentárias nos respectivos segmentos foi a seguinte: começar pelas superficies vestibulares, continuar pelas superfícies oclusais ou incisais e terminar com as superficies linguais. A escova era colocada em posição inclinada sobre as superficies vestibulares e linguais, a partir do colo do dente, escovando os dentes superiores para baixo e os inefriores para cima. As superficies oclusais foram escovadas em movimento de diante para trás e de trás para diante. 
CASTELLANOS, R.A. Estudo comparativo do efeito de solução de NaF a $0,5 \%$, através de bochecho, escovação e ambos, na prevenção da cárie dental. Rev. Saúde públ., S. Paulo, $17: 461-75,1983$.

Antes e após a escovação das superfícies de cada segmento, a escova era embebida na solução de $\mathrm{NaF}$, distribuida num copo individual para cada criança. Os escolares que fizeram, além da escovação, os bochechos, receberam uma quantidade adicional de $10 \mathrm{ml}$. da solução para bochecharem após a escovação.

Para facilitar a supervisão da aplicação da solução de $\mathrm{NaF}$ por meio da escovação foram chamados, de cada vez, grupos de $\mathbf{5}$ crianças.

As crianças iniciaram a aplicação do bochecho e da escovação em setembro de 1975; durante os meses de dezembro do mesmo ano, e janeiro, fevereiro e julho de 1976, não o fizeram devido às férias escolares; em novembro de 1976, isto é, após 10 meses de efetiva aplicação da solução de $\mathrm{NaF}$ a $0,5 \%$ foi realizado um segundo levantamento da experiência de cárie nessas crianças.

Análise dos resultados: nesta apresentação não serão analisados os resultados observados nas superfícies dentárias separadamente (presentes no início e as que irromperam durante o estudo). Foram consideradas como componentes do estudo apenas as crianças presentes ao primeiro e segundo exame e que realizaram pelo menos vinte auto-aplicações com a solução de flúor. Foi feito também reajuste de reversões (superfície cariada no 10 exame e hígida no 2o exame) e quando um dente foi extraido entre o primeiro e segundo exame, a superficie foi considerada com o mesmo valor que recebeu no primeiro levantamento.

As comparaçōes de médias foram feitas mediante análise de variância paramétrica e contraste de Sheffé ${ }^{1 \mathbf{4}, \mathbf{5} 0}$, aceitando-se as considerações de Glass e col.24, julgando que os dados estavam razoavelmente próximos às condições expostas por tal autor. O nivel de significância adotado foi de 0,05 . Quando evidenciada uma redução significativa entre as médias de um grupo experimental e a de controle foi calculada a redução percentual estabelecendo-se 0 respectivo intervalo de confiança (Fieller-nivel de $95 \%)^{5,16,10}$.

\section{RESULTADO E DISCUSSAO}

Durante a realização do estudo houve uma perda de 117 crianças, correspondendo a $27,6 \%$ em relação às 424 crianças que iniciaram o estudo, das quais $90(21,2 \%)$ foi devido a transferência de escola principalmente no inicio do ano escolar de 1976. As restantes $27(6,4 \%)$ abandonaram o estudo porque ou os pais argumentaram que os filhos sentian-se mal após as aplicações de flúor, ou as crianças recusaram-se a comparecer ao tratamento, ou porque não completaram um mínims de 20 aplicações durante o estudo.

Ao calcular novamente o CPO-S médio e a média de superficies irrompidas das crianças que iniciaram e completaram o estudo, estabeleceu-se uma nova composição dos grupos (Tabela 2) que a análise estatística indicou não deferirem significantemente entre sí (Tabelas 3 e 4); estes valores também não foram significantemente diferentes dos valores do levantamento original (Tabela 1).

Pelos dados apresentados na Tabela 5 , pode-se verificar que a média de superfícies permanentes irrompidas foi similar para os 4 grupos de estudo, confirmado estatisticamente ao nivel de 0,05 (Tabela 7 ).

Nota-se, também, na Tabela 5 , que os 3 grupos experimentais apresentam valores médios CPO-S menores que o grupo controle, diferenças estas que em termos percentuais são apresentadas na Tabela 6 . Analisados os incrementos entre os grupos experimentais e controle (Tabela 8), verificou-se que os mesmos são diferentemente significantes ao nivel de $0,05 \mathrm{e}$, as realizar-se o teste de contrastes, essas diferenças corresponderam aos 3 grupos experimentais em relação ao grupo controle, não indicando 
CASTELlaNOS, R.A. Estudo comparativo do efeito de solução de NaF a 0,5\%, através de bochecho, escovação e ambos, na prevenção da cárie dental. Rev. Saúde públ., $\mathbf{s}$. Pauln. $17: 461-75,1983$.

diferença significante entre os grupos experimentais entre si (Tabela 9).

Pode observar-se, tabém pela (Tabela 5), que o coeficiente de ataque de cárie foi maior para o grupo controle do que para os grupos experimentais, e que o grupo IV apresentou o menor coeficiente de ataque.

Os resultados do intervalo de confiança indicam que a redução percentual de cárie obtida através dos diferentes métodos de aplicação da solução de flúor não deveria ser menor do que $15,91 \%$ e não maior do que $58,05 \%$ (Tabela 6 ).

Comparando os valores de redução de cárie obtidos no presente estudo com os obtidos por outros autores, com soluções em concentração e aplicações diferentes, observaram-se resultados similares aos obtidos com o Grupo IV. Assim, o valor

\section{T A B E L A 1}

Idade média (anos), média de superficies irrompidas, CPO-S e coeficiente de ataque de cárie, das 424 crianças que iniciaram o estudo, segundo os grupos experimentais e controle. São Paulo, 1975.

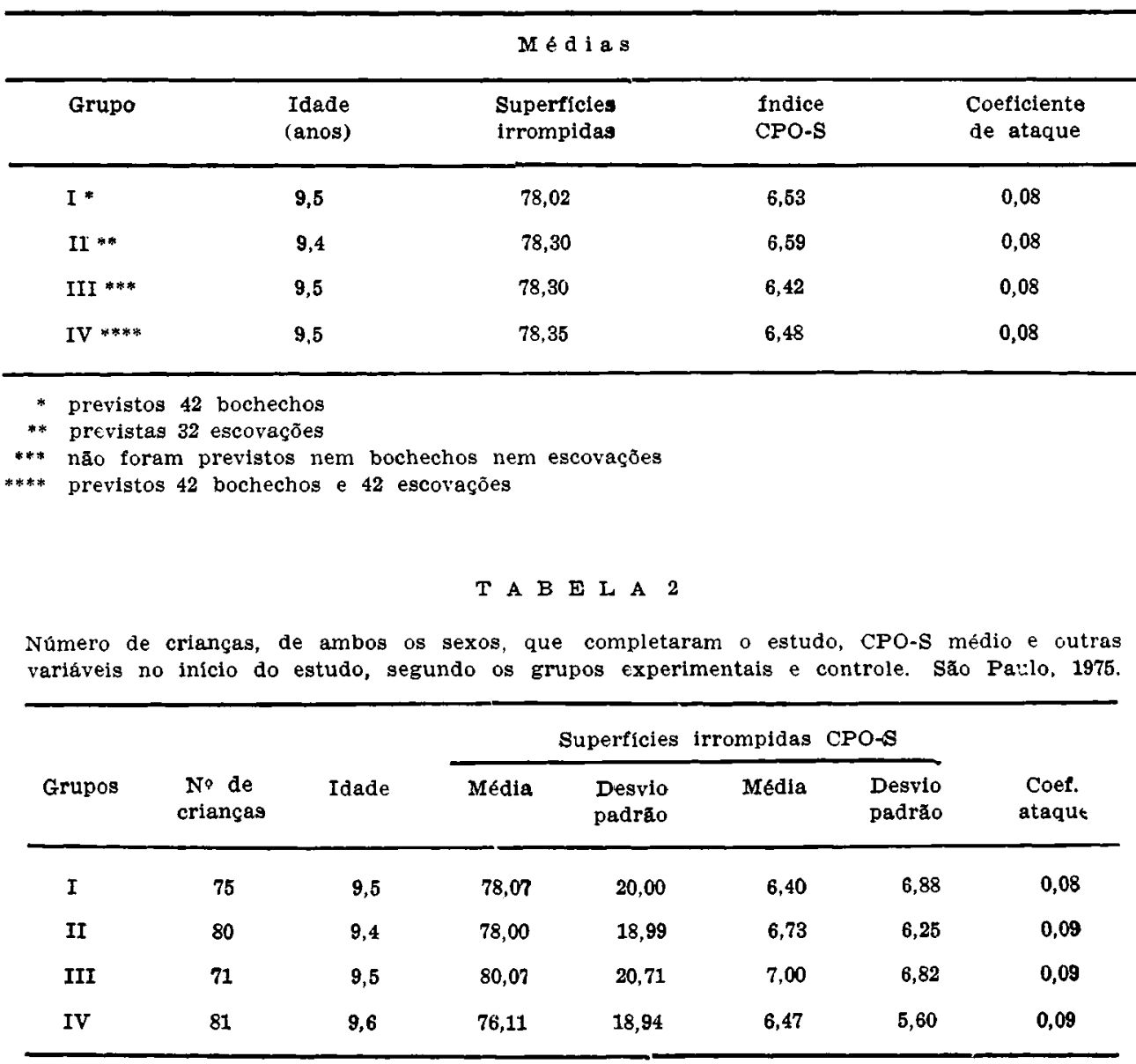




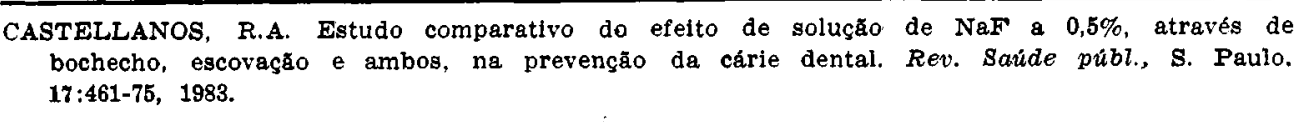

T A B E L A 3

Análise de variância para os valores médios de superfícies irrompidas das 307 crianças, de ambos os sexos que completaram o estudo, no início do tratamento, segundo os grupos experimentais e controle. São Paulo, 1975.

\begin{tabular}{|c|c|c|c|c|}
\hline $\begin{array}{l}\text { Fontes de } \\
\text { Variagão }\end{array}$ & $\begin{array}{c}\text { Graus de } \\
\text { Liberdade }\end{array}$ & $\begin{array}{l}\text { Soma de } \\
\text { Quadrados }\end{array}$ & $\begin{array}{l}\text { Quadrado } \\
\text { Médio }\end{array}$ & $F$ \\
\hline $\begin{array}{l}\text { Entre } \\
\text { tratamentos }\end{array}$ & 3 & 593,6822 & 197,8941 & $\begin{array}{r}0,5134 \\
p>0,1\end{array}$ \\
\hline $\begin{array}{l}\text { Dentro de } \\
\text { tratamentos }\end{array}$ & 308 & $116.799,3146$ & 385,4763 & (năo significante) \\
\hline Total & 306 & $117.392,9968$ & - & - \\
\hline
\end{tabular}

T A B E L A 4

Análise de variância para os valores médios CPO-S das 307 crianças de ambos os sexos que completaram o estudo, no início do tratamento, segundo os grupos experimentais e controle. São Paulo, 1975.

\begin{tabular}{lcccc}
\hline $\begin{array}{c}\text { Fontes de } \\
\text { Variação }\end{array}$ & $\begin{array}{c}\text { Graus de } \\
\text { Liberdade }\end{array}$ & $\begin{array}{c}\text { Soma de } \\
\text { Quadrados }\end{array}$ & $\begin{array}{c}\text { Quadrado } \\
\text { Médio }\end{array}$ & F \\
\hline $\begin{array}{l}\text { Entre } \\
\text { tratamentos }\end{array}$ & 3 & 16,4635 & $\mathbf{5 , 4 8 7 8}$ & $\begin{array}{r}0,1347 \\
\mathbf{p}>0,1\end{array}$ \\
$\begin{array}{l}\text { Dentro de } \\
\text { tratamentos }\end{array}$ & 303 & $12.348,1228$ & $\mathbf{4 0 , 7 5 2 9}$ & (não significante) \\
\hline Total & 306 & $\mathbf{1 2 . 3 6 4 , 5 8 6 3}$ & - & - \\
\hline
\end{tabular}

de $49,77 \%$ de redução da cárie dentária obtido no Grupo IV (Tabela 6) é similar aos relatados por Torell e Ericsson ${ }^{55}$, de $49,1 \%$ após 2 anos de bochechos diários com NaF a $0,05 \%$ em crianças de 10 anos de Idade; aos de Moreira e Tumang ${ }^{40,41}$, de $51,13 \%$ e $46,8 \%$ de redução do índice CPO-S, após 1 e 2 anos de execução de bochechos com NaF a $0,1 \%$ numa frequiência de 3 vezes por semana, aos de Horowitz e col.27 de $44 \%$ de redução do indice CPO-S, após 20 meses de utilização de um bochecho semanal com $\mathrm{NaF}$ a $0,2 \%$; aos de Soto $\mathrm{P}$. e
Maiwald 51 de $46 \%$ de redução do índice C.PO-S, após 28 meses de aplicação de um bochecho quinzenal com $\mathrm{NaF}$ a $0,2 \%$ em crianças de 6 a 7 anos de idade e uma redução de cárie de $43,4 \%$ CPO-S no grupo de crianças de 10 e 11 anos de idade, após 88 meses de duração do programa (Maiwald e Soto P. ${ }^{35}$ ), e de Torell e Siberg ${ }^{57}$ de 48,7\%, $51,9 \%$ e 53,5 de redução do indice CPO-S, após um ano de bochechos mensais com $\mathrm{NaF}$ a $0,2 \%$ em grupos de crianças de 9 anos de idade. Naturaimente que todos esses resultados são superiores aos do pre- 
CASTEllaNos, R.A. Estudo comparativo do efeito de solução de NaF a $0.5 \%$, através de bochecho, escovação e ambos, na prevenção da cárie dental. Rev. Saúde públ., S. Paulo. $17: 461-75,1983$.

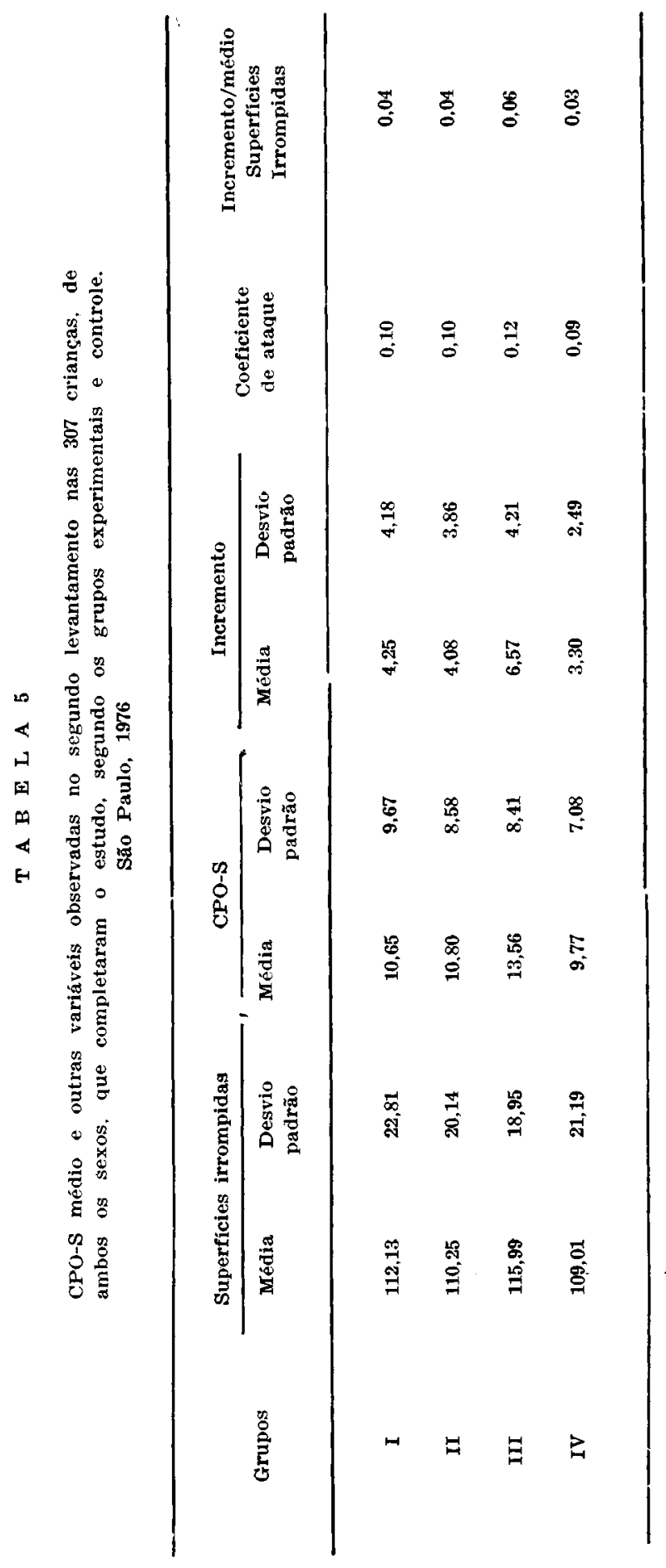


CASTELlaNOS, R.A. Estudo comparativo do efetto de solução de NaF a $0,5 \%$, através bochecho, escovação e ambos, na prevenção da cárie dental. Rev. Saúde públ., S. PauI 17:461-75, 1983.

T A B E L A 6

Percentual de red:ção de cárie dental e intervalo de confiança para os grupos que foram significantemente diferentes no incremento de cárie em relação ao grupo controle. Săo Paulo, 1976.

\begin{tabular}{rcc}
\hline Grupos & \% de Reduçăo & $\begin{array}{c}\text { Intervalo de confiança }-95 \% \\
\%\end{array}$ \\
\hline I e III & 35,19 & $15,91|-| 43,49$ \\
II e III & 37,91 & $19,34|-| 45,96$ \\
IV e III & 49,77 & $32,99|-| 58,05$ \\
\hline
\end{tabular}

$\mathrm{s}-=13,87$

T A B E L A 7

Análise de variância para os valores médios de superfícies irrompidas das 307 crianças, d: cimbos os sexos, que completaram o estudo, segundo os grupos experimentais e controle. São Paulo, 1976.

\begin{tabular}{lcccc}
\hline Fontes de variação & $\begin{array}{c}\text { Graus de } \\
\text { Liberdade }\end{array}$ & $\begin{array}{c}\text { Soma de } \\
\text { Quadrados }\end{array}$ & $\begin{array}{c}\text { Quadrado } \\
\text { Médio }\end{array}$ & F \\
\hline $\begin{array}{l}\text { Entrc } \\
\text { tratamentos }\end{array}$ & 3 & $2.071,5585$ & 690,5195 & $\begin{array}{c}1,5898 \\
\mathbf{p}>0,1\end{array}$ \\
$\begin{array}{l}\text { Dentro de } \\
\text { tratamentos }\end{array}$ & 303 & $131.605,6402$ & 434,3420 & (não significante) \\
\hline Total & 306 & $133.677,1987$ & - & - \\
\hline
\end{tabular}

T A B E L A 8

Análise de variáncia para os valores médios de superficies irrompidas das 307 crianças, de ambos os sexos, que completaram o estudo, segundo os grupos experimentais e controle. São Paulo, 1976.

\begin{tabular}{lcccc}
\hline $\begin{array}{c}\text { Fontes de } \\
\text { Variação }\end{array}$ & $\begin{array}{c}\text { Graus de } \\
\text { Liberdade }\end{array}$ & $\begin{array}{c}\text { Soma de } \\
\text { Quadrados }\end{array}$ & $\begin{array}{c}\text { Quadrado } \\
\text { Médio }\end{array}$ & F \\
\hline $\begin{array}{l}\text { Entre } \\
\text { tratamentos }\end{array}$ & 3 & 438,6198 & 146,2066 & $\begin{array}{r}10,5375 \\
\text { p }<0,001\end{array}$ \\
$\begin{array}{l}\text { Dentro de } \\
\text { tratamentos }\end{array}$ & 303 & $4.204,0903$ & 13,8749 & (significante) \\
\hline Total & 306 & $4.642,7101$ & - & - \\
\hline
\end{tabular}


CASTELlaNos, R.A. Estido comparativo do efeito de solução de NaF a 0,5\%, através de bochecho, escovação e ambos, na prevenção da cárie dental. Rev. Saúde públ., S. Paulo, $17: 461-75,1983$.

\section{T A B E L A 9}

Resultados dos testes de Sheffé para os valores médios do incremeento CPO-S das 307 crianças de ambos os sexos, que completaram o estudo, segundo os grupos experimentais e controle. São Paulo, 1976.

\begin{tabular}{|c|c|c|c|}
\hline Grupos & II & III & IV \\
\hline I & $\begin{array}{c}0,2980 \\
p>0,1 \\
\text { (não significante) }\end{array}$ & $\begin{array}{r}-3,7580 \\
0,01<p<0,025 \\
\text { (significante) }\end{array}$ & $\begin{array}{c}1,6057 \\
p>0.1 \\
\text { (não significante) }\end{array}$ \\
\hline & II & $\begin{array}{c}-4,1042 \\
0.005<\mathrm{p}<0.01 \\
\text { (significante) }\end{array}$ & $\begin{array}{c}1,3277 \\
p>0,1 \\
\text { (não significante) }\end{array}$ \\
\hline & & III & $\begin{array}{c}\quad 5,4082 \\
0,001<\mathrm{p}<0,005 \\
(\text { significante })\end{array}$ \\
\hline
\end{tabular}

sonte estudo quando se leva em consideração o Grupo 1 que apenas bochechou ou 0 Grupo Il que somente fez escovações.

Como se pode observar pelas comparações realizadas, os resultados são similares quando se usa a solução de NaF em diferentes concentrações, frequiencias e períodos de aplicação, o que aparentemente indica que parece existir determinado limite de redução do ataque de cárie, além do qual a frequiência e a concentação da soltção não atuam mais; pode-se supor que haja uma saturação de flúor no esmalte o qual, conseqüentemente, não absorveria o flúor que posteriormente venha a ser administrado. Aceitando-se estas suposições como verdadeiras, seria então mais aconselhável utilizar concentrações mais baixas e em maior frequiência, como a recomendada por Torell e Ericsson 55, ou seja, de $0,05 \%$ para uso diário; ou de $0,025 \%$ com freqüência semanal, que, em contato com o esmalte produz um composto estável (Forsman ${ }^{20}$ ).

Dever-se-ia, pelas dificuldades de aplicações freqüentes sptar-se pela freqüência semanal ou quinzenal, na concentração de $0,2 \%$ (Horowitz ${ }^{27}$; Soto P. e Maiwald 51), sendo recomendável que se realizem outros estudos com a concentração de $0,025 \%$ que, ao que tudo indica, alcança os mesmos resultados (Forsman ${ }^{20}$ ) e parece possuif as qualidades desejáveis para uma solução de flúor para bochecos (Torell e Ericsson 54).

A não existência de diferenças significantes entre os métodos de escovação e de bochechos, evidenciadas neste estudo, vem corroborar as afirmaçōes de Torell e Ericsson ${ }^{54,56}$ e de Rosenkranz ${ }^{47}$, que não encontraram diferenças significativas entre bochechos e escovações com uma solução de $\mathrm{NaF}$ a $0,5 \%$ aplicada mensalmente $e$ por um periodo de 3,5 anos pelo primeiro, e $\mathrm{NaF}$ a $0,2 \%$ aplicada quinzenalmente $\mathrm{e}$ po: um periodo de um ano pelo segundo.

A redıção da cárie obscrvada na presente pesquisa $(37,91 \%)$, utilizando-se o método de escovação (média de 37,6 aplicações-ano) é superior à redução de cárie obtida por Bergren e Wellander ${ }^{4}$ com uma solução mais concentrada ( $1 \%$ ), com apenas 5 escovaçōes no primeiro ano e 4 escovações no segundo ano, ou seja, de 25 a $30 \%$ menos CPO-S, redução percentual esta que foi confirmada pelos mesmos autores ${ }^{3}$ quando obtiveram, com uma solução a $0,5 \%$, na mesma frequiência e período de estudo, uma reduçãn de $29 \%$ - CPO-S.

Entretanto Marthaler ${ }^{37}$ e Marthaler e Mulhermann 28 obtiveram reduçōes que variavam de 30 a $50 \%$ menos CPO-S, com quatro a seis escovações supervisionadas 
CASTELlanOS, R.A. Estudo comparativo do efeito de solução de NaF a $0,5 \%$, através de bochecho, escovação e ambos, na prevenção da cárie dental. Rev. Saúde públ., S. Paulo, 17:461-75, 1983 .

por ano. $O$ benefício total, no entanto, não pode ser atribuído exclusivamente às escovações com o composto de flúor, pois o programa preventivo era mais amplo, compreendendo a utilização de sal com flúor, comprimidos de flúor, escovação supervisionada e educação em saúde bucal, e ainda dispondo no mercado, para comercialização, de dentifricio com flúor.

Estes fatos demonstram as dificuldades de se comparar os resultados deste estudo, obtidos por meio da utilização de bochechos ou de escovaçōes, com os estudos realizados por outros autores. A comparação torna-se mais complexa e difícil devido a existência de diferenças quanto à freqüência de aplicação, quanto à concentração e tipo de solução empregada, quanto ao tempo de estudo, idade das crianças, e atendimento dentário, quanto à análise, avaliaçăo e apresentaçăo dos resultados, e quanto aos efeitos combinados de agentes preventivos, entre outros fatores, diferenças estas parcialmente evidenciadas nas comparaçōes entre os estudos iá mencionados.

Esta problemática realmente suscita várias indagações e, consequientemente, o desejo de realizar novos estudos entre os quais, por exemplo, o de testar a mesma solução na mesma concentração, com o mesmo tempo de exposição na cavidade bucal e mesma forma de administração, mas com diferentes frequiências de aplicação e em vários grupos da mesma população, a fim de se estabelecer o minimo de frequiência com o qual poder-se-ia chegar ao mesmo resultado.

$\mathrm{Em}$ relação às reversōes ocorridas, verifica-se pela Tabela 10 , que o maior número correspondeu às crianças do grupo IV, seguidas pelas do grupo I e III as reversões nos grupos experimentais talvez possam ser melhor explicadas em funçāo da remineralizaçāo que ocorre após a aplicação de flưor e que às vezes pode retardar ou mesmo interferir no processo de desmineralização do dente, em especial em lesões incipientes tornando higida a superfície que anteriormente teria sido diagnosticada como cariada; contudo não se tem a mesma explicação para o grupo III, o qual não esteve exposto à ação do flưor. Portanto, estas reversões devem ser aceitas como sendo um processo normal de erros de diagnóstico esperado dentro da normalidade de variabilidade $e$ que, no caso presente, não foram além de $2,7 \%$ em relação ao total de observaçōes ou de $3,5 \%$ em relação às observações no grupo IV.

$\mathrm{Na}$ Tabela 2 observa-se que os coeficientes de ataque de cárie no início do estudo são similares para os 4 grupos, e depen-

T A B E L A 10

Número, média e percentual de ocorrência de reversóes de diagnóstico em superfícies dentárias atacadas pela cárie no primeiro exame nas 307 crianças, de ambos os sexos, que completaram o estudo, segundo os grupos experimentais e controle. São Paulo, 1976.

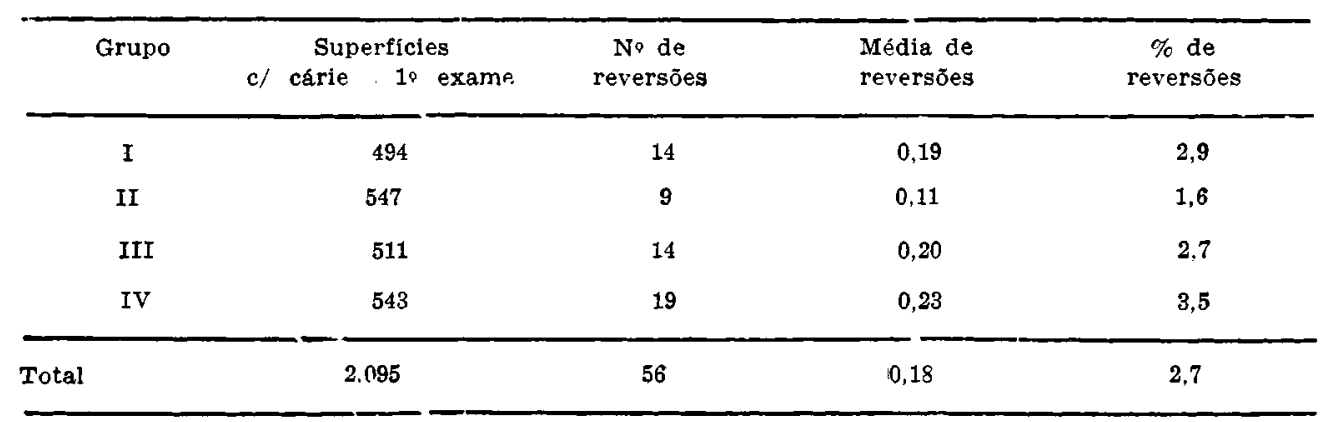


CASTELlaNOS, R.A. Estudo comparativo do efeito de solução de NaF a $0,5 \%$, através de bochecho, escovação e ambos, na prevenção da cárie dental. Rev. Saúde públ., S. Paulo. 17:461-75, 1983.

dendo se liouve ou não proteção das superfícies contra o ataque de cárie, a diferença de valores entre os grupos experimentais e controle se distancia mais, o que aliás era de esperar-se (Tabela 5). Provavelmente este coeficiente de ataque de cárie poderia servir no futuro, como um indicador simples e rápido para avaliar ataques de cárie em crianças com diferente número de superfícies irrompidas, ao invés de comparar apenas as médias de ataque de cárie em grupos populacionais. Também poderia ser utilizado para a constituição inicial dos grupos de estudo de pesquisa com agentes preventivos e talvez até, dependendo de um maior número de observações, estabelecer se existe alguma relação entre valores do coeficiente e significância de redução ao aumento do ataque de cárie.

Com base na literatura consultada, parece que se pode concluir que o $\mathrm{NaF}$ neutro é a solução mais comprovadamente indicada para a aplicação de bochechos e/ou escovações, como procedimento preventivo contra a cárie dental. Ainda que as soluções ácidas de flúor apresentem um efeito similar ao obtido com as soluções neutras, elas possuem a desvantagem do sabor desagradável (mais difícil de ser mascarado) e a necessidade de serem preparados em laboratórios (o que aumenta os custos). Estes argumentos também são válidos para o fluoreto de estanho que ainda tem a desvantagem de instabilidade e de provocar manchas nas superfícies restauradas com silicato ou com resinas.

Outro aspecto que deve ser salientado refere-se à continuidade da aplicação da solução de flúor, que deve ser ininterrupta, acompanhando o desenvolvimento do individuo, isto é, desde a época em que o dente irrompeu até pelo menos 5 anos de exposição na cavidade bucal, periodo em que o dente apresenta maior susceptibilidade ao ataque de cárie (Carlos e Gittelsohn ${ }^{12}$ ). Pensando na dentição permanente como um todo, estes métodos deveriam ser utilizados até a idade de 16 a 17 anos, dependendo da época de erupção do segundo molar permanente, pois ficou demonstrado pelas observações de Koch 32 que, quando cessa a aplicação do procedimento (bochechos), a superfície do dente torna-se novamente susceptivel ao ataque de cárie. Este argumento da persistência na aplicação da solução de flúor, também encontra suporte nos resultados apresentados por Maiwald e Soto P. ${ }^{35}$ para a população escolar cubana (aproximadamente 1 milhão de crianças beneficiadas) em que foi evidenciada uma diminuição constante nos valores médios dos indices CPO e CPO-S, e a inversão dos componentes desses índices, após 7 anos de aplicação quinzenal contínua com a solução $\mathrm{NaF}$ a $0,2 \%$. Resultados semelhantes são referidos por Torell ${ }^{53}$, Torell e Ericsson ${ }^{54}$ e Bikerland e col.8, para a população escolar dos países escandinavos em que, após 10 e mais anos de aplicação da solução de flúor, com freqüência quinzenal e acompanhada por outras medidas de prevenção e de educação em saúde bucal, os benefícios tornaram-se similares aos alcançados pela fluoretação das águas de abastecimento púbilico. Esses benefícios foram evidenciados pela diminuição dos dentes indicados para extração, e pela redução do número de dentes atacados pela cárie, bem como pela diminuição do tempo gasto por tratamento completado. Outro benefício foi o aumento de cobertura, dando atendimento a número maior de crianças com os mesmos recursos existentes e até aumentando o tipo de serviço oferecido à população escolar. Apoiados na análise estatística pode-se concluir que:

1. Houve uma redução significante do ataque de cárie para ambos os métodos de aplicação da solução de $\mathrm{NaF}$ a $0,5 \%$ com freqüência semanal (bochecho $\mathrm{e}$ escovaçāo, separadamente e em forma combinada) em relação ao grupo controle;

2. Não houve diferenças significantes entre as reduçōes de cárie obtidas por meio dos diferentes procedimentos de aplicação da solução de flúor. 
CASTELLANOS, R.A. Estuuo comparativo do efeito de solição de NaF a $0,5 \%$, através de bochecho, escovação e amhos, na prevençâo da cárie dental. Rev. Saúde públ., S. Paulo, 17: $461-75,1983$.

Cumpre ser ressaltado que, embora estatisticamente os efeitos não foram diferentes, no caso de vir a ser utilizada uma solução de $\mathrm{NaF}$ a $0,5 \%$, seria recomendável, pelos resultados desta pesquisa, como método de aplicação, o uso combinado de escovação e bochechos preferencialmente ao de bochecho e escovação tm separado, pelas seguintes razōes:

\section{a) Pela análise de custo-beneficio}

A redução do ataque de cárie, em valores percentuais (análise global) entre os grupos 1 e lll de $35,19 \%$ CPO-S, ll e lll de $37,91 \%$ e IV e IIl de 49,77 , levaria desde o ponto de vista administrativo, a uma economia significativa quando estes valores são projetados para grupos populacionais. Para esta estimativa levou-se em consideração os seguintes dados:

- a população de 1975 nas idades de 10 a 11 anos, com base nos dados de Pinto ${ }^{44}$ e a população de 10 a 14 anos, para 1980, utilizando a projeção populacional elaborada por Santos 49 ;

- o custo do tratamento por dente de Cr $\$ 64,19$ (Pinto ${ }^{* 4}$ ), acrescido de $40 \%$ pela inflação anual durante os 4 anos (1976 a 1979);

- o ataque de cárie $\mathrm{C}+\mathrm{Ei}$ de 4,10 (Pinto ${ }^{44}$ ) cujos valores foram transformados em superfícies, usando a regressão de Knutson ${ }^{31}$;

- o custo por bochecho-ano, baseado no custo de Cr\$ 4,72 proporcionado pela DENPAO* em 1979, ao qual se acrescentou $\operatorname{Cr} \$ 0,10$ pela diferença da quantidade de sal necessária para a nossa concentração de $0,5 \%$;

- o custo de escovação-ano, baseado no custo da solução de $\mathrm{NaF}$ a $0,5 \%$, e no custo da escova dental de cerca de Cr\$ 15,00 cada escova para uma média de 40 escovações-ano).
A partir desses dados chega-se aos seguintes valores:

- um total de 5.629 .928 crianças nas idades de 10 e 11 anos para 1980 ;

- um C + Ei médio de 7,22;

- um custo de Cr\$ 140,00 por superficie tratada (US\$ 3.20);

- um custo de Cr\$ 4,82 por bochecho-ano (US\$ 0.11 );

- um custo de Cr\$20,00 por escovaçāo-ano (US\$ 0.46).

De posse destes cálculos, estimou-se a economia de custos, que representaria a redução de cárie para as crianças do grupo I (bochechos) e do grupo IV (escovação e bochecho), chegando aos seguintes resultados: para o grupo 1, com percentual de redução de $35,19 \%$, resultaria numa economia de Cr\$ 1.933.622.831,00, ou seja, quase 2 bilhōes de cruzeiros; para o grupo IV, com percentual de redução de cárie de $49,77 \%$ resultaria numa economia de Cr\$ 2.427.666.000,00, ou seja, quase 2 bilhões e 500 milhões de cruzeiros (US\$ $55,565,713.00$ ).

\section{b) Pelo valor educativo.}

Possibilitaria oferecer um reforço contínuo da importância da escovação, fazendo com que as crianças adquirissem esse hábito de higiene bucal que proporciona, quando utilizado de forma correta e na freqüência adequada, proteção dos dentes e dos tecidos periodontais.

Caso as dificuldades sejam de tal magnitude que não permitam a implantação do programa combinado de escovação e bochecho, a nossa escolha recairia num programa de bochechos por haver maior facilidade em sua implantação, se bem que uma menor economia que a do método anterior estimada em Cr\$ 494.043.169,00 (aproximadamente 500 milhōes de cruzeiros a menos) (US\$ $11,307,466.00)$.

* Informação pessoal fornecida, em 15-10-79 por Antonio G.F. Rosa, Chefe da equipe técnica de estudos e normas da Divisão de Estudos, Normas e Programas de Assistência ao Escolar (DENPAO). 
CASTELI_ANOS, R.A. Estudo comparativo do efeito de soltção de NaF a $0,5 \%$, através de bochecho, escovação e ambos, na prevenção da cárie dental. Rev. Saúde públ., S. Paulo, 17:461-75, 1983.

\section{CONCLUSŌES}

Após terem sido analisados estatisticamente os resultados da utilização de uma solução de $\mathrm{NaF}$, semanalmente na concentração de $0,5 \%$ sob a forma de bochechos, escovação ou ambos em escolares de 9 a 10 anos de idade, parece lícito concluir que a redução percentual de $35,19 \%$ (Grupo I), $37,91 \%$ (Grupo II) e $49,77 \%$ (Grupo IV) foi estatisticamente significante em relação ao grupo controle (Grupo III), ao nivel de 0,05 ; e que a redução percentual quando comparados os grupos experimentais entre si não foram estatisticamente significante ao nível de 0,05.

CASTELLANOS, R. A. [A comparative study of the effect of a $0.5 \%$ NaF solution, used in mouthwashing or toothbrushing or both, in the prevention of dental caries]. Rev. Saúde públ. S. Paulo, 17:461-75, 1983.

ABSTRACT: A comparative study of the anticariogenic effect of sodium fluoride solution, with a concentration of $0.5 \%$, applied weekly and under supervision, in mouthwashing and toothbrushing, used independently and in combination, was carried out on 424 children of 9 and 10 years of age from five public schools in S. Paulo, Brazil, over a period of 10 school months. The children were equally divided into three experimental groups and one control group according to the dental age (number of erupted permanent teeth surfaces) and history of caries (DMF-S). Three hundred and seven children were examined at the end of the study and the results show a statistically significant decrease at the 0.05 level, between the experimental groups and control group, in the incidence of caries $-35.19 \%$ for the mouthwashing group (Group I); $37.91 \%$ for the toothbrushing group (Group II); and $49.77 \%$ for the mouthwashing and toothbrushing group (Group IV) - on the surfaces present at the beginning and the surfaces that erupted during the study. When the experimental groups were compared there was no signicant difference in proportions at the 0.05 level. t.

UNITERMS: Dental caries, prevention. Fluorine. School children.

\section{REFERENCIAS BIBLIOGRAFICAS}

1. AASENDEN, $R$. et al. Effects of daily rising and ingestion of fluoride solutions upon dental caries and enamel fluoride. Arch. oral Biol., $17: 1705-14,1972$.

2. BENNIE, A. et al. Five years of community preventive dentistry and health education in the country of Sutherland, Scotland. Community Dent. oral Epidem., 6:1-5, 1978.

3. BERGGREN, H. \& WELANDER; E. The carie inhibiting effect of sodium ferric and zirconium fluorides. Acta odont. scand., $22: 401-13,1964$

4. BERGGREN, H. \& WELANDER. E. Superrised tooth brushing with a sodium fluoride solution in 5,000 sweedish schoolchildren: results and analysis of procedures. Acta odont. scand., 18:209-34, 1960.

5. BERQUó, E.S. \& MARQUES, R.M. Análise de variáncia. São Paulo, Faculdade de Higiene e Saúde Pública USP. Departamento de Estística, 1963.

6. BIBBY, B.G. et al. Preliminary reports on the effect on dental caries of the use of sodium fluoride in a prophylactic cleanning mixture and on a mouthwash. J. dent. Res., 25:207-11, 1946.

7. BIRKELAND, J.M. et al. An approach to objective assessment of caries prophy- 
CASTEllanos, R.A. Estudo comparativo do efeito de solução de NaF a $0,5 \%$, através de bochecho, escovação e ambos, na prevenção da cárie dental. Rev. Saúde públ., S. Paulo, 17:461-75, 1983.

lactic measures in a dental health program. Community Dent. oral Epidem., 3:126-31, 1975 .

8. BIRKELAND, J.M. et al. Benefits and prognoses following 10 years of a fluoride mouthrinsing program. $J$. dent. Res., 85:31-7, 1977.

9. BRAMS, N.V. Preventive dentistry in the scandinavian school dental health service. Int. dent. J., 17:384-92, 1967.

10. BULLEN, D.C.T. et al. One year effect of supervised tooth brushing with an acidulated fluoride-phosphte solution. $J$. Eanad. dent. Ass., 31:231-5, 1965.

11. BULLEN, D.C.T. et al. Two years effect of supervised tooth brishing with an fluoride-phosphate solution. $J$. Canad. dent. Ass., 32:89-93, 1966.

12. CARLOS, J.P. \& GITTELSOHN, A. M. Longitudinal studies of the natural history of caries. II - A life-table study of caries incidence in the permanent teeth. Arch. oral Biol., 10:739-51, 1965.

13. CONCHIE, J.M. et al. Three years of supervised tooth brushing with a fluoride-phosphate solution. $J$. publ. Hith Dent., 29:11-8, 1969.

14. COSTA NETO, P.L. de O. Estatistica. São Paulo, Ed. Edgard Blucher, 1977.

15. DE PAOLA, P.F. et al. Effect of high-concentration ammonium and sodicm fluoride rinses on dental caries in schoolchildren. Community Dent. oral Epidem., 5:7-14, 1977.

16. DUBLEY, S.D. On the determination of confidence limits of an index. Biometrics, 22 :603-9, 1966.

17. FACULDADE DE SAŨdE PÚBLICA da USP. Disciplina de Odontologia Sanitária. Critérios para exames e regras para - registro dos indices CPO e CPOS. São Paulo, 1967. [mimeografado]

18. FINN, S.B. et al. The chemical cariostatic effectiveness of two concentrations of acidulated phosphate-fluoride mouthwash. J. Amer. dent. Ass., 90:398-402, 1975.

19. FINNEY, D.J. Statistical method in biological assay. 2nd ed. London, Griffin, 1971.

20. FORSMAN, B. The caries preventing effect of mouthrinsing, with $0.025 \%$ sodium fluoride solution in sweedish children. Community Dent. oral Eprdem., 2:58-65. 1974.
21. FRANKL, S.N. et al. The topical anticariogenic effect of dally rinsing with an acidulated phosphate-fluoride solution. J. Amer. dent. Ass., $85: 882-6,1972$.

22. GALLAGHER, S.J. et al. Self-application of fluoride by rinsing. $J$. publ. Hlth Dent., 34:13-21, 1974 .

23. GERDIN, P.O. \& TORELL, P. Mouth rinses with potassium fluoride solutions containing manganese. Caries Res., 3:99-107, 1969.

24. GLASS, R.L. et al. The appropriatness of analysis of variance to the statistical analysis of dental clinical trials. Arch. oral Biol. 17:633-43, $19: 2$.

25. HEIFETZ, S.B. et al, The effect on dental caries of weekly rinsing with a neutral sodium fluoride or an acidilated phosphate-fluoride mouthwash. J. Amer. dent. Ass., 87:364-8, 1973.

26. HEIFETZ, S.B. et al. Two years evaluation of a self-administered procedure for the topical application of acidulated phosphate-fluoride: final report. $J$. publ. Hlth Dent., 30:7-12, 1970.

27. HOROWITZ, H.S. et al. The effect on human dental caries of weekly oral rinsing with a sodium fluoride mouthwash: a final report. Arch. oral Biol,, $16: 609-16,1971$.

28. HOROWITZ, H.S. et al. Evaluation of self-administered prophylaxies and supervised tooth brishing with acidulated phosphate-fluoride. Caries Res., 8:39-51, 1974.

29. IRMISCH, V.B. et al. Karies-prophylaxe durch uberwachte Mundpülaktionen Ergebnisse nach 4 jähriger Kontrollizeit. Zahn-, Mund-u Kieferheilk, 62:690-3, 1974.

30. KLEIN, H. Studies on dental caries. I Dental status and dental needs of clementary schoolchildren. Publ. Hlth Rep., $53: 751-65,1938$.

31. KNUTSON, J.W. Epidemiological trends patterns of dental caries prevalence data. J. Amer. dent. Ass., 57:821-9, 1958.

32. KOCH, G. Caries increment in schoolchildren during and two years after end of supervised rinsing of the mouth with sodium fluoride solution. Odont. Revy, 20:323-30, 1969.

33. KOCH, G. Effect of sodium fluoride in dentifrice and mouthwash on incidence of dental caries in schoolchildren. Odont. Revy, 18 (suppl.) 1967. 
CASTEllaNos, R.A. Estudo comparativo do efeito de solução de NaF a $0,5 \%$, através de bochecho, escovação e ambos, na prevenção da cárie dental. Rev. Saúde públ., s. Paulo, $17: 461-75,1983$.

34. McCONCHIE, J.M. et al. Caries preventive effect of two concentrations of stannous fluoride mouth rinse. Community Dent. oral Epidem., 5:278-83, 1977.

35. MAIWALD, V.H.J. SOTO PADRON, F. Ergebnisse der Kollektiven kariesprävention durch Mundspulungen mit $0,2 \%$ iger Natriumfluoridlösung nach 88 Monaten. Stomatol. $D D R, 27: 835-40,1977$.

36. MARTHALER, T.M. Estimation of simple size for longitudinal clinical caries trials. Helv. odont. Acta, 22:168-73, 1967.

37. MARTHALER, T.M. Reduction of caries, gingivitis and calculus after eight years of preventive measures observations in seven communities. Helv. Odont. Acta, $16: 69-83,1972$.

38. MARTHALER, T.M. MUHLEMANN, H. R. Clinical anticaries effect of supervised toothbrushing with fluoride tablets for topical application. Helv. odont. Aota, 14:77-9, 1970.

39. MOREIRA, B.W. Estudo sobre os efeltos de bochechos com solução molar de fluoreto de sodio, na prevenção da cárie dental. Piracicaba, 1970. [Tese de Doutoramento - Faculdade de Odontologia de Piracicaba da UNICAMP]

40. MOREIRA, B.W. \& TUMANG, A.J. Bochechos com solução de fluoreto de śdio a $0,1 \%$ na prevenção da cárie dental. Rev. bras. Odont., 28:11-9, 1971.

41. MOREIRA, B.W. \& TUMANG, A.J. Prevenção da cárie dentária através de bochechoś com soluçōes de fluoreto de sódio a $0,1 \%$ : resultados após 2 anos de estudos. Rev. bras. Odont., 29:37-42, 1972.

42. OLLINEN, P. Munskőljning eller borstning med olika fluoridlösningar. Sverig. Tandläk. Förb. Tidn., 58:913, 1966. Apud Torell, P. ER Ericsson, Y.56

43. ORGaNizaço MUNDIAL DA SAODE. Grupo Científico 'sobre Etiologia $y$ Prevencion de la Caries Dental, Ginebra, 1971. Informe. Ginebra, 1972, (Ser. inf. tec., 494).

44. PINTO, V.G. Caracteristicas do subsistema público federal de prestação de serviços em odontologia. Brasilia, DF, 1977. [Dissertação de Mestrado - Faculdade de Saúde Pública da USP].
45: RADIKE, A.W. et al. Clinical evaluation of stannous fluoride as an anticaries mouthrinse. J. Amer. dent. Ass., $86: 404-8,1973$.

46. ROBERTS, J.F. et al. The effect of an acidulated fluoride mouthwash on dental caries. J. dent. Res., 27:497-500, 1948.

47. ROSENKRANZ, F. Karies prophylaktischer Vergleich $\mathbf{Z}$ wis chen mundspülen und Zahneputzen mit Natriumfluorid Lösung. Odont. T., 75:528-34, 1967.

48. RUGG-GUNN, A.J. et al. Caries prevention by daily fluoride mouthrinsing: report of a three year clinical trial. Brit. dent. J., $135: 353-60$, 1973.

49. SANTOS, J.L.F. Projeção da população brasileira: 1970-2000. Rev. Saúde públ., S. Paulo, 8 (supl): 91-102, 1974.

50. SCHEFFE, H. A method for judging all contrast in the analysis of variance. Biometrika, 40:87-104, 1953.

51. SOTO PADRON, F. \& MAIWALD, H.J. Analisis de los resultados de las aplicaciones topicas de flioruros a grupos de poblacion en Cuba. Rev. cuba. Estomat., $10: 173-8,1973$.

52. SWERDLOFF, G. \& SHANNON, I.L. Feasibility of the use of stannous fluoride mouthwash in a school system. J. Dent, Child., 36:363-8, 1969.

53. TORELL, $P$, The Goteborg studies of methods for applying fluorides topically. Adv. Fluorine Res. dent. Caries Prev., $3: 255-8,1964$.

54. TORELL, P, ERICSSON, Y. Beneficios potenciales de los colutorios con fluoruros. Washington, D.C., Departamento de Servicios de Salud, Oficina Sanitaria Panamericana, 1975. (Doc. ne HP/DH/40) [Mimeografado].

55. TORELL, P. \& ERICSSON, Y. Two year clinical test with different methods of local - preventive fluorine application in swedish school children. Acta odont. scand., 23:287-322, 1965.

56. TORELL, P. \& ERICSSON, $Y$. The value on caries prevention of methods for applying fluorides topically to the teeth. Int. dent. J., 17:564-81, 1967.

67. TORELL, P. \& SIBERG, A. Mouthwash with sodium fluoride and potassium fluoride. Odont. Revy, 13:62-72, 1962.

Recebido para publicação em 23/06/1983 Aprovado para publicasão em 01/09/198s 